\title{
Oro-pharyngeal chemoreceptor activation induces gastric motor response in healthy volunteer subjects
}

\author{
Chiharu AsHIDA ${ }^{1}$, Akira KoJIMA ${ }^{2}$, \\ Motoi KoBASHI ${ }^{3}$ and Tomoshige KoGA ${ }^{1}$ \\ ${ }^{1}$ Department of Rehabilitation, Kawasaki University of Medical Welfare, Kurashiki 701- \\ 0193, Japan \\ ${ }^{2}$ Handa Surgery Hospital, Aioi 678-0031, Japan \\ ${ }^{3}$ Department of Oral Physiology, Okayama University Graduate School of Medicine and \\ Dentistry, Okayama 700-8525, Japan
}

\begin{abstract}
Previous study has revealed that water-responsive afferent neurons in the superior laryngeal nerve induced inhibition of motility in the proximal and distal stomach using anaesthetized rats. These gastric responses might facilitate the reservoir function of the stomach. To confirm the gastric responses discovered in rats also occur in humans, we evaluated gastric myoelectrical activities in healthy volunteer subjects during fluid intake using electrogastrography. Before human experiments, we recorded the myoelectrical activities in rats to evaluate the response induced by the administration of water into the larynx. A large deflection in the gastric myoelectrical activities was observed just after the administration of water in anesthetized rats. A similar large deflection was also observed just after voluntary swallowing of $20 \mathrm{ml}$ water in humans. The swallowing of saliva did not induce such response. We further observed the gastric response during reflex swallowing elicited by slow infusion of isotonic saline, water or $0.05 \mathrm{M}$ citric acid on to the posterior tongue. Infusion of water and citric acid but not $0.15 \mathrm{M}$ saline induced significant changes in mean relative ratio of the response. These electrogastrographic responses induced by the infusion of liquids strongly suggest that the gastric motor response facilitates reservoir function of the stomach during liquid intake in humans as well as in rats.
\end{abstract}

Key words: stomach, electrogastrography, superior laryngeal nerve, swallowing

\section{Introduction}

The afferent neurons in the superior laryngeal nerve (SLN), which bifurcates from the vagal nerve, are responsible for protective reflexes such as gagging (Davies et al., 1995; Fukuda and Koga, 1997), apnea (Storey and Johnson, 1975) and reflex swallowing (Storey, 1968). The SLN, which innervates taste buds distributed on the laryngeal surface of the epiglottis and on the larynx, responds to mechanical and chemical stimulation of the larynx and epiglottis (Storey and

Correspondence to: Tomoshige Koga, Ph. D, Department of Rehabilitation, Kawasaki University of Medical Welfare, Kurashiki 701-0193, Japan

Phone: +81-86-462-1111 Fax: +81-86-464-1109 e-mail: koga@mw.kawasaki-m.ac.jp 
Johnson, 1975). Individual fibers of the SLN are broadly chemosensitive as are other gustatory nerves (Bradley et al., 1983; Smith and Hanamori, 1991). The most obvious difference between the sensitivities of chemosensitive fibers of the SLN and those in other gustatory nerves is in the response to water (Bradley et al., 1983; Harada and Smith, 1992; Smith and Hanamori, 1991). Several studies have suggested that afferent neurons in the SLN responding to water are involved in important functions, such as diuresis, prandial drinking and cardiovascular responses (Hanamori and Ishiko, 1993; Miyaoka et al., 1987; Shingai et al., 1988).

In addition, previous studies revealed that water-responsive afferent neurons in the SLN induced inhibition of gastric motility using anaesthetized rats (Kobashi et al., 2002; Kobashi et $a l ., 2000)$. Water-responsive neurons in the SLN suppress phasic contractions in the distal stomach and induce gastric relaxation in the proximal stomach via vagal efferent nerves. These responses prevented intragastric pressure from becoming high during ingestion. Therefore, those might facilitate the reservoir function of the stomach during liquid intake. However, it has not been well clarified whether these gastric responses occur in humans. Electrogastrography is a recording of the myoelectrical activity of the stomach by means of electrodes placed on the abdominal skin surface (Alvarez, 1922). The electrogastrography is noninvasive way to evaluate gastric motility. It has been widely used in healthy humans (Chen et al., 1994) and in patients (Gad-el-Hak and Bakr, 2001) to evaluate gastric motility. Thus, electrogatrography is useful to evaluate gastric motor activities in humans.

The present study aimed to demonstrate that the gastric motor response discovered in rats is useful to facilitate reservoir function during liquid intake in humans. We recorded gastric myoelectrical activities in healthy volunteer subjects to evaluate gastric motor response during voluntary swallowing and reflex swallowing.

\section{Methods}

Twenty-one healthy volunteers (age $25.2 \pm 0.98$ years, 4 female and 17 male) participated in this study. All of them gave their informed consents to be enrolled in this study. All experimental procedures were performed in accordance with the Ethics Committee of Kawasaki Medical School.

All subjects were asked to relax their muscles in a silent room in which the air temperature was adjusted to $\sim 25^{\circ} \mathrm{C}$ with a relative humidity of $\sim 40 \%$. Gastric myoelectrical activities were recorded from two cutaneous bipolar disposable $\mathrm{Ag} / \mathrm{AgCl}$ electrodes (Fukuda Denshi, Japan). One electrode was placed midway between the umbilicus and xiphoid, and the other was placed in the midclavicular line below the left costal margin. Each electrode was placed after wiping the surface of the skin with ethanol. The myoelectrical activities were amplified by a modified biophysical amplifier (AB621G, Nihon Kohden, Japan). A bandpass filter was set to within the range of 0.02 to $0.5 \mathrm{~Hz}$. To evaluate the time course of swallowing, laryngeal movements were measured using force transducer attached on the thyroid cartilage. All data were stored using a magnetic tape recorder and converted to digital data using an analog-to-digital converter (Power Lab, AD Instruments).

Gastric myoelectrical activities were recorded during voluntary swallowing in 8 subjects. 
Before swallowing test solution, subjects were asked to swallow their saliva. After the recording during swallowing saliva, they were asked to swallow $20 \mathrm{ml}$ water at a stretch. After $5 \mathrm{~min}$ rest, they were asked to swallow $20 \mathrm{ml}$ of $0.15 \mathrm{M}$ saline at a stretch. To evaluate the effects of reflex swallowing on the gastric response, infusion tests were performed in 13 subjects. Each subject lied on his back at an angle of 45 degree. The tip of a silicon tube (OD: $5 \mathrm{~mm}$ ) was positioned on the posterior tongue of each subject to infuse test solutions. Test solutions were delivered at a rate of $0.5 \mathrm{ml} / \mathrm{sec}$ for $30 \mathrm{sec}$ using peristaltic pump. Water, $0.15 \mathrm{M}$ saline and $0.05 \mathrm{M}$ citric acid were delivered in each subject.

Before human experiments, we preliminary observed gastric myoelectrical activities using urethane-chloralose anaesthetized rats. Bipolar platinum spherical electrodes were placed on the abdominal muscle, one was placed at $1 \mathrm{~cm}$ lateral and the other was placed $1 \mathrm{~cm}$ caudal to processus xiphoideus. The responses induced by the administration of water $(0.1 \mathrm{ml})$ into the larynx are shown in Fig. 1A. The method to infuse test solution was mentioned previously (Kobashi et al., 2000). A large deflection of the wave was observed just after the administration of water. Therefore, we used this extraordinary deflection as an index of the response. For normalizing data, ratio between the amplitude $1 \mathrm{~min}$ before swallowing and that just after swallowing was used as a relative ratio to analyze the responses in human. All numerical values are represented as means \pm SEM. Significant differences were evaluated using Student's $t$-test $(P<0.05$ for significance).

\section{Results}

Mean peak frequency of myoelectrical activities obtained from subjects was $3.07 \pm 0.12$ $(n=21)$ showing normal electrical rhythm of the human stomach. As shown in Fig. 1Ba, no obvious change in the electrical activities was observed during swallowing of saliva. On the other hand, the intake of water induced large deflection (Fig. 1Bb) as well as the response observed in rats (Fig. 1A). Mean relative ratio is shown in Table 1. Mean relative ratio induced by water intake was significantly larger than that induced by swallowing of saliva $(t=2.95, P<0.05$, $\mathrm{n}=8$ ). Similar responses were observed during the intake of isotonic saline (Table 1). Mean relative ratio was not significantly different with that induced by swallowing of saliva $(t=1.58$, NS, $n=8$ ). However, significant difference was not observed between the mean relative ratio induced by water intake and that induced by isotonic saline intake $(t=0.73, \mathrm{NS}, \mathrm{n}=8)$. Therefore, we could not exclude the possibility that the responses during water intake were induced by mechanoreceptors situated in the pharynx or esophagus.

The electrogastrographic responses during reflex swallowing induced by slow infusion of test solution were observed. Figure $1 \mathrm{C}$ shows the myoelectrical activities during the infusion of 3 test solutions. The infusion of isotonic saline did not induce obvious change in the activities, although reflex swallowing was observed (Fig. 1Ca). The infusion of water induced rapid change in amplitude of the response (Fig. 1Cb). The infusion of citric acid had stronger effects. (Fig. $1 \mathrm{Cc})$. Mean relative ratio clearly showed that both water $(t=2.29, P<0.05, \mathrm{n}=13)$ and citric acid $(t=2.38, P<0.05, \mathrm{n}=13)$ induced significant change as compared with isotonic saline (Table 1$)$. In addition, the latency of the first swallow and the number of swallows during the infusion were 

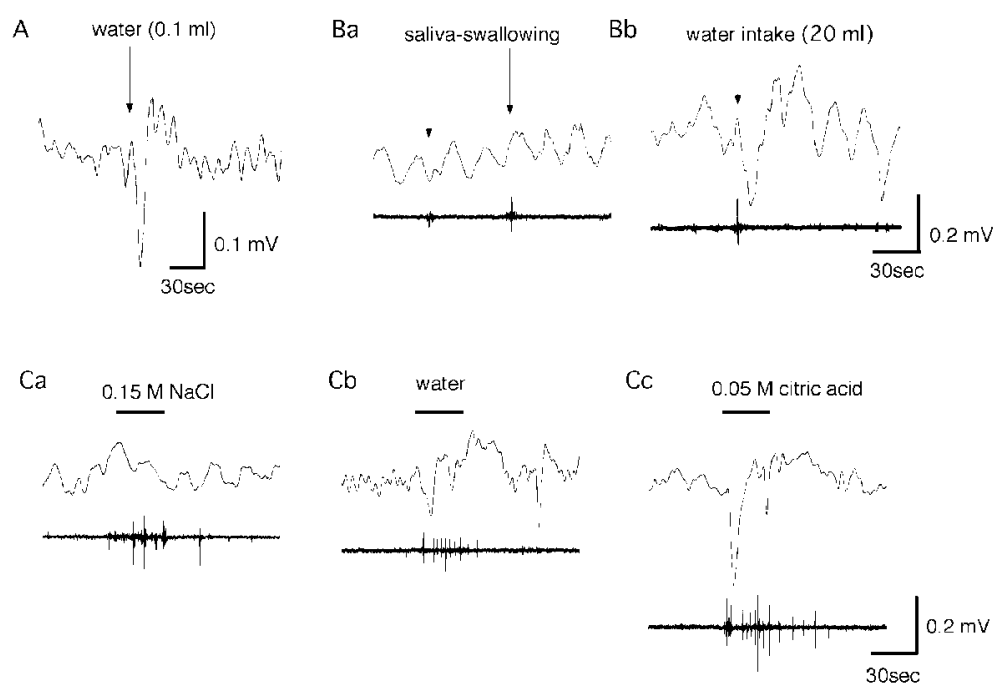

Fig. 1. Typical electrogastrographic response during swallowing in rat (A) and in human (B and C). A: response of myoelectrical activities induced by the administration of water into the larynx in a urethane-chloralose anaesthetized rat. B: myoelectrical activities during voluntary swallowing in human. Swallowing saliva (a) did not induce obvious change, but swallowing of water (b) induced large deflection as well as the response observed in rats. Upper trace showed gastric myelectrical activity. Lower trace showed the laryngeal movements corresponding to swallowing. C: myoelectrical activities during reflex swallowing induced by the infusion of indicated solutions in human. Although isotonic saline did not induce obvious change, the infusion of either water or citric acid induced phasic deflections during reflex swallowing. Gastric myoelectrical activity (upper trace) and the laryngeal movements (lower trace) are showed.

Table 1 Summary of responses observed in human EGG during voluntary swallowing and reflex swallowing

\begin{tabular}{|c|c|c|c|}
\hline & $\begin{array}{l}\text { Mean relative } \\
\text { ratio of EGG }\end{array}$ & $\begin{array}{l}\text { Latency of the first } \\
\text { swallowing (sec) }\end{array}$ & $\begin{array}{c}\text { Number of swallowings } \\
\text { (count } / 30 \mathrm{sec})\end{array}$ \\
\hline \multicolumn{4}{|c|}{ Voluntary swallowing $(n=8)$} \\
\hline Saliva & $1.02 \pm 0.05$ & - & - \\
\hline $0.15 \mathrm{M} \mathrm{NaCl}$ & $1.41 \pm 0.24$ & - & - \\
\hline Water & $1.64 \pm 0.20^{*}$ & - & - \\
\hline \multicolumn{4}{|c|}{ Reflex swallowing ( $\mathrm{n}=13$ ) } \\
\hline $0.15 \mathrm{M} \mathrm{NaCl}$ & $1.06 \pm 0.05$ & $14.0 \pm 0.75$ & $3.6 \pm 0.33$ \\
\hline Water & $1.37 \pm 0.13^{\star *}$ & $10.8 \pm 1.33$ & $4.4 \pm 0.39$ \\
\hline $0.05 \mathrm{M}$ citric acid & $1.58 \pm 0.21^{\star *}$ & $8.9 \pm 0.89^{* *}$ & $5.5 \pm 0.25^{\star *}$ \\
\hline
\end{tabular}

${ }^{*}$ significant difference compared with mean relative ratio when saliva was swallowed. ${ }^{* *}$ significant differences compared with mean relative ratio, latency of the first swallow and number of swallows when isotonic saline was infused.

measured. Both the latency of the first swallow ( $t=3.33, P<0.05, \mathrm{n}=13)$ and the number of swallows $(t=4.12, P<0.05, \mathrm{n}=13)$ during the infusion of citric acid was significantly different, compared with those induced by the infusion of isotonic saline. 


\section{Discussion}

The results obtained in the present research revealed that the gastric motor response found in rats also occur in humans suggesting the activation of oro-pharyngeal chemoreceptors induces gastric motor response in human. Receptive relaxation is one of the reflexes caused by the extrinsic nervous system during swallowing and facilitates the reservoir function of the stomach (Cannon and Lieb, 1911). It was suggested that this inhibitory response of the stomach is caused by mechanical stimulation of the pharynx and esophagus by ingesta (Abrahamsson and Jansson, 1969). The present study strongly suggested that oro-pharyngeal chemoreceptive afferents, in addition to the mechanoreceptors in the pharynx and esophagus, are a neuronal source for the receptive relaxation.

In the present study, we analyzed the electrogastrographic responses based on the amplitude of the responses observed just after swallowing. Preliminary experiments using rats revealed that a large deflection of wave was induced just after the administration of water into the larynx. In the previous study (Kobashi et al., 2000), similar injections of water into the larynx immediately induced the inhibition of phasic contractions in the distal stomach and did relaxation in the proximal stomach. Therefore, we should consider that a large deflection appeared just after swallowing corresponds with the inhibition of gastric motility observed in the previous study. Since a large deflection was also observed when each volunteer subject swallowed water, we used the amplitude of this large deflection as an index of the gastric motor response.

Because that the obvious change in the myoelectrical activities was not observed during voluntary swallowing of saliva, we considered the swallowing movement itself did not induce gastric response. Voluntary intake of water induced the large deflection in the myoelectrical activities. Therefore, liquid intake induced gastric motor response. However, voluntary swallowing of isotonic saline induced un-negligible change. Thus, we considered that mechanoreceptive afferents as well as chemoreceptive ones induced the gastric response analyzing the myoelectrical activities during voluntary swallowing of relatively larger volume of liquid. To further investigate the contribution of chemoreceptive afferents themselves to the gastric response, we used slow infusion of test solutions. Slow infusion of test solutions induced reflex swallowing. The infusion of water but not isotonic saline induced the large response in the myoelectrical activities. The infusions of citric acid induced larger response than that induced by the infusion of water. Thus, the magnitude of gastric motor response during reflex swallowing depends on the quality of test solutions.

Taste buds are especially concentrated on the laryngeal surface of the epiglottis (Belecky and Smith, 1990; Bradley et al., 1980; Miller and Smith, 1984). Taste buds in this region appear to be ideally situated for protecting the airway during accidental aspiration of food or fluid. However, in the present study, subjects did not show protecting reflex such as cough or gagging. The aryepiglottal folds and the upper esophagus also contain taste buds in addition to the laryngeal surface of the epiglottis (Belecky and Smith, 1990; Miller and Smith, 1984; Travers and Nicklas, 1990). Taste buds situated in these regions would be stimulated during normal fluid intake to induce gastric motor response. 
The affferent neurons in the pharyngeal branch of the glossopharyngeal nerve (GPN-ph) showed the similar response profile with the SLN afferents, that is, the neurons in the GPN-ph respond to sour taste in addition to water (Hanamori and Ishiko, 1993). Electrical stimulation of the GPN-ph also induced swallowing in rats (Kitagawa et al., 2002). The electrophysiological properties of the GPN-ph to induce swallowing were not different with those of the SLN. The GPN-ph afferents are supposed to facilitate the elicitation of swallowing with the SLN afferents in rats (Kajii et al., 2002). Therefore, the contributions of the GPN-ph, in addition to the SLN, to the gastric response observed in the present research are considered. The glossopharyngeal afferents, in collaboration with the SLN afferents, might induce the gastric motor response in humans.

\section{Acknowledgement}

This research was supported in part by Project Research Grants of Kawasaki University of Medical Welfare to T.K.

\section{References}

Abrahamsson, H. and Jansson, G. (1969). Elicitation of reflex vagal relaxation of the stomach from pharynx and esophagus in the cat. Acta Physiol. Scand. 77: 172-178.

Alvarez, W.C. (1922). The electrogastrogram and what it shows. J. Am. Med. Ass. 78: 1116-1119.

Belecky, T.L. and Smith, D.V. (1990). Postnatal development of palatal and laryngeal taste buds in the hamster. J. Comp. Neurol. 293: 646-654.

Bradley, R.M., Mistretta, C. and Kim, Y. (1980). Quantitative analysis of developping epiglottal taste buds in sheep. J. Anat. 130: 25-32.

Bradley, R.M., Stedman, H.M. and Mistretta, C.M. (1983). Superior laryngeal nerve response patterns to chemical stimulation of sheep epiglottis. Brain Res. 276: 81-93.

Cannon, W.B. and Lieb, C.W. (1911). The receptive relaxation of the stomach. Am. J. Physiol. 29: 267273.

Chen, J.D., Richards, R.D. and McCallum, R.W. (1994). Identification of gastric contractions from the cutaneous electrogastrogram. Am. J. Gastroenterol. 89: 79-85.

Davies, A.E., Stone, S.P. and MacMahon, J. (1995). Pharyngeal sensation and gag reflex in healthy subjects. Lancet 345: 487-488.

Fukuda, H. and Koga, T. (1997). Neuronal gagging activity patterns may be generated by neurons in the reticular area dorsomedial to the retrofacial nucleus in dogs. Exp. Brain Res. 113: 394-401.

Gad-el-Hak, N. and Bakr, A.M. (2001). Gastric myoelectrical activity in diabetics with and without diabetic autonomic neuropathy. Hepatogastroenterol. 48: 590-593.

Hanamori, T. and Ishiko, N. (1993). Cardiovascular responses to gustatory and mechanical stimulation of the nasopharynx in rats. Brain Res. 619: 214-222.

Harada, S. and Smith, D.V. (1992). Gustatory sensitivities of the hamster's soft palate. Chem. Senses 17: $37-51$.

Kajii, Y., Shingai, T., Kitagawa, J., Takahashi, Y., Taguchi, Y., Noda, T. and Yamada, Y. (2002). Sour taste stimulation facilitates reflex swallowing from the pharynx and larynx in the rat. Physiol. Behav. 77: 321-325.

Kitagawa, J., Shingai, T., Takahashi, Y. and Yamada, Y. (2002). Pharyngealbranch of the glossopharyngeal nerve plays a major role in reflex swallowing from the pharynx. Am. J. 
Physiol. 282: R1342-R1347.

Kobashi, M., Koga, T., Mizutani, M. and Matsuo, R. (2002). Suppression of vagal motor activities evokes laryngeal afferent-mediated inhibition of gastric motility. Am. J. Physiol. 282: R818-R827.

Kobashi, M., Mizutani, M. and Matsuo, R. (2000). Water stimulation of the posterior oral cavity induces inhibition of gastric motility. Am. J. Physiol. 279: R778-R786.

Miller, I. Jr. J. and Smith, D.V. (1984). Quantitative taste bud distribution in the hamster. Physiol. Behav. 32: 275-285.

Miyaoka, Y., Sakaguchi, T., Yamazaki, M. and Shingai, T. (1987). Changes in water intake following pharyngolaryngeal deafferentation in the rat. Physiol. Behav. 40: 369-371.

Shingai, T., Miyaoka, Y. and Shimada, K. (1988). Diuresis mediated by the superior laryngeal nerve in rats. Physiol. Behav. 44: 431-433.

Smith, D.V. and Hanamori, T. (1991). Organization of gustatory sensitivities in hamster superior laryngeal nerve fibers. J. Neurophysiol. 65: 1098-1114.

Storey, A.T. (1968). Laryngeal initiation of swallowing. Exp. Neurol. 20: 359-365.

Storey, A.T. and Johnson, P. (1975). Laryngeal water receptors initiating apnea in the lamb. Exp. Neurol. 47: 42-55.

Travers, S.P. and Nicklas, K. (1990). Taste bud distribution in the rat pharynx and larynx. Anat. Rec. 227: 373-379.

(Received September 1, 2004; Accepted September 14, 2004) 